Fluoroacetate and fluorocitrate have been isolated from soy beans grown in high inorganic fluoride media, indicating the possibility that edible plants are the source of covalently bonded fluoride ${ }^{6}$.

I thank Mrs Joy Howe and Mr C. F. Ning for technical assistance. This work was supported by a grant from the National Institutes of Health, US Public Health Service.

Department of Pharmacology,

Donald R. Taves

University of Rochester,

School of Medicine and Dentistry,

New York.

Received August 16; revised October 10, 1968.

${ }^{1}$ Taves, D. R., Nature, 217, 1050 (1968).

2 Taves, D. R., Talanta,15, 969 (1968).

${ }^{3}$ Taves, D. R., Talanta (in the press).

' Carlson, C. H., Armstrong, W. D., and Singer, L., Amer. J. Physiol., 199,

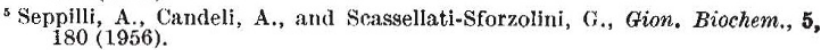
'Cheng, J. Y., Yu, M. H., Miller, G. W., and Welkie, G. W., Env. Sci. Tech., 2,
367 (1968).

\section{Optical Activity and the Fixation of Rods and Cones}

Vertebrate rod outer segments have been known for more than a century to rotate the plane of polarization of transmitted light ${ }^{1}$. When the polarizer in the path of a beam illuminating a microscope stage points north, and the analyser crosses it, suspensions of fresh bleached rods and cones obtained from the retinae of the frog (Rana esculenta), cat, rabbit and goldfish severally show that the receptors pointing along a NW axis maximally transmit light independently of the colour of the illuminant. Some of the conditions which effect a switch of the axis of maximum transmission through $90^{\circ}$ have been discussed by Schmidt ${ }^{1}$, an extension of whose work is now under way (unpublished results of G. M. Villermet and myself), and has been briefly reported ${ }^{2}$. This preliminary report draws attention to the differential action of two fixatives, namely formaldehyde and glutaraldehyde.

Three parts from the same frog retina were fixed in formaldehyde (4 per cent $\mathrm{w} / \mathrm{v}$ in $200 \mathrm{mM} \mathrm{KHPO} / \mathrm{K}_{2} \mathrm{PO}_{4}$ buffer, $p \mathrm{H} 7 \cdot 0)$, dilute formaldehyde $(0.8$ per cent $\mathrm{w} / \mathrm{v}$ in $200 \mathrm{mM} \mathrm{KHPO}_{4} / \mathrm{K}_{2} \mathrm{PO}_{4}$ buffer, $p \mathrm{H} 7 \cdot 0$ ), and glutaraldehyde $(2.5$ per cent $\mathrm{w} / \mathrm{v}$ buffered in sodium cacodylate, $0.1 \mathrm{M}, p \mathrm{H} .7 .3)$ respectively. Both formaldehyde samples showed marked NW (that is, normal) activity within 30 min of the onset of fixation and $2 \mathrm{~h}$ later. After about $6 \mathrm{~h}$, activity changed to NF. In the case of glutaraldehyde, there was no rod showing NW (that is, normal) activity either within $30 \mathrm{~min}$ of the onset of fixation or $2 \mathrm{~h}$ later: but there was marked activity in a direction perpendicular to that shown in normal suspensions and those recently fixed in formaldehyde. Similar differences between the fixatives were observed with receptors of the other species. Attention has already been drawn ${ }^{2}$ to parallels between the effects of these solutions on optical activity and the production of the early receptor potential ${ }^{3}$.

There is also a remarkable difference between the relative rates of fixing rods and cones respectively. Although this can be studied on material obtained from frogs, goldfish retinae facilitate the observations because their cones are large. If a goldfish retina is fixed with glutaraldehyde, the change in the direction of the optical activity of the rods, from NW to NE, is instantaneous. But the change in the cones occurs only 30-60 min later. While formaldehyde acts more slowly on the goldfish retina than does glutaraldehyde-as we saw to be true of the frog-it acts more slowly on the cones of the fish than on its rods.
This difference in the rates with which fixatives change optical activity in rods and cones respectively is surprising. Cohen $^{4}$ has advanced evidence for the view that the cone membrane invaginates many times and intercepts the disks carrying the visual pigment. Disks and mernbrane are probably in contact with each other. On the other hand, rods seem to consist more nearly of membranous bags with isolated disks inside. If the membranes of rods and cones are equally permeable to the fixatives, then the change in birefringence accompanying fixation would be expected to occur more rapidly in cones than in rods. But the reverse is observed, so that one may conclude tentatively that the membranes are not equally permeable to these fixatives.

\section{R. A. Wfalle}

Institute of Ophthalmology,

London WC1.

Received September 30, 1968

1 Schmidt, W. J., Kolloidzeitschrift, 85, 137 (1938).

${ }^{2}$ Weale, R. A., Proc. Fifth Intern. Cong. Photobiol. (in the press).

${ }^{8}$ Arden, G. B., Bridges, C. D. B., Ikeda, H., and Siegel, I. M., Vision Res. 8, 3 (1968).

${ }^{4}$ Cohen, A., J. Cell. Biol., 37, 424 (1968).

\section{Hunter--Hurler Syndrome : Gel Filtration and Dialysis of Urinary Acid Mucopolysaccharides}

Patients with Hunter-Hurler syndrome, which is an inborn error of metabolism, have excessive deposition of acid muccpolysaccharides (AMPS) in various organs ${ }^{1,2}$, and large amounts of chondroitin sulphate $B$ and/or heparitin sulphate are produced in the urine ${ }^{3}$. In contrast, normal people excrete a small amount of AMPS, chiefly in the form of chondroitin sulphate $C$ and chondroitin sulphate $A$. The determination of urinary AMPS is being used extensively in the study and in the diagnosis of this disease and of its several genetic variants.

We report here the results of our studies on gel filtration and dialysis of the urinary AMPS of patients with this condition and of normal controls. Urinary AMPS were isolated from non-dialysed $24 \mathrm{~h}$ urine specimens with the Ecteola (chloride form) column method of DiFerrante ${ }^{4}$ or by precipitation with ettyl pyridinium chloride. A column $0 \cdot 6 \times 86 \mathrm{~cm}$, packed with 'Sephadex $G-200$ ', was used for the gel filtration of the urinary AMPS. Elution was performed with $0.025 \mathrm{M} \mathrm{NaCl}$, at room temperature and at a flow rate of $6.5 \mathrm{ml} / \mathrm{h}$. Fractions of urinary AMPS obtained by chromatography on 'Dowex 1-X 2' (CI) were used for the calibration of the column. The molecular weights of these fractions were determined by a combined sedimentation-diffusion method.

A considerable amount of AMPS isolated from the urine of Hunter-Hurler patients dialysed easily through the boiled or unboiled cellulose dialysis tubing (size 27, wall thickness 0.001 inches, Union Carbide Corporation) (Table 1). Up to 50 per cent of the AMPS was lost during three successive dialyses: first against distilled water, then against $1.0 \mathrm{M} \mathrm{NaCl}$ and finally against distilled water. In contrast, only 11 per cent of the urinary AMPS from normal controls was lost in the same conditions of dialysis. The loss of heparitin sulphate from Hunter-Hurler patients during dialysis in regular Visking casing has been reported $^{5}$. It is interesting that the carbazole-orcinol ratio of the dialysable AMPS (patient R. V.) in three successive dialyses was $0.78,1.00$ and 1.03 respectively, compared with 0.54 for the non-dialysable material and 0.69 for the non-dialysed sample. This shows that in addition to a considerable loss of AMPS during dialysis, there is an alteration in the ratio of the remaining individual components of the AMPS. The dialysis of the untreated urine against distilled water showed a significant 